\title{
TOPOLOGICAL TRIVIALITY OF LINEAR DEFORMATIONS WITH CONSTANT LE NUMBERS
}

\author{
Christophe Eyral and Maria Aparecida Soares Ruas
}

\begin{abstract}
Let $f(t, \mathbf{z})=f_{0}(\mathbf{z})+\operatorname{tg}(\mathbf{z})$ be a holomorphic function defined in a neighbourhood of the origin in $\mathbf{C} \times \mathbf{C}^{n}$. It is well known that if the one-parameter deformation family $\left\{f_{t}\right\}$ defined by the function $f$ is a $\mu$-constant family of isolated singularities, then $\left\{f_{t}\right\}$ is topologically trivial - a result of A. Parusiński. It is also known that Parusiński's result does not extend to families of non-isolated singularities in the sense that the constancy of the Lê numbers of $f_{t}$ at $\mathbf{0}$, as $t$ varies, does not imply the topological triviality of the family $\left\{f_{t}\right\}$ in general - a result of J. Fernández de Bobadilla. In this paper, we show that Parusiński's result generalizes all the same to families of nonisolated singularities if the Lê numbers of the function $f$ itself are defined and constant along the strata of an analytic stratification of $\mathbf{C} \times\left(f_{0}^{-1}(0) \cap g^{-1}(0)\right)$. Actually, it suffices to consider the strata that contain a critical point of $f$.
\end{abstract}

\section{Introduction}

Let $U$ be an open neighbourhood of the origin in $\mathbf{C}^{n}(n \geq 2), D$ an open disc about the origin in $\mathbf{C},(t, \mathbf{z}):=\left(t, z_{1}, \ldots, z_{n}\right)$ linear coordinates for $\mathbf{C} \times \mathbf{C}^{n}$, and

$$
f:(D \times U, D \times\{\mathbf{0}\}) \rightarrow(\mathbf{C}, 0), \quad(t, \mathbf{z}) \mapsto f_{t}(\mathbf{z}):=f(t, \mathbf{z}),
$$

a holomorphic function. (This notation implies $f(t, \mathbf{0})=0$ for all $t \in D$.) We assume that the dimension of the critical locus $\Sigma f_{t}$ of $f_{t}$ at $\mathbf{0}$ is $\geq 0$ (i.e., $f_{t}$ has a critical point at $\mathbf{0})$ and constant as $t$ varies. As usual, we write $V\left(f_{t}\right)$ for the hypersurface $f_{t}^{-1}(0)$ defined by the function $f_{t}$. In [9], Lê Dũng Tráng and C. P. Ramanujam showed that if the one-parameter deformation family $\left\{f_{t}\right\}$ defined by $f$ is a $\mu$-constant family of isolated singularities and if, furthermore, $n \neq 3$, then the corresponding family of hypersurfaces $\left\{V\left(f_{t}\right)\right\}$ is topologically trivial.

2010 Mathematics Subject Classification. 32S15, 32S25, 32S05.

Key words and phrases. Hypersurface singularity; linear deformation; Lê numbers; Thom's $a_{f}$ condition; topological triviality.

Partially supported by CNPq grants \# 305651/2011-0 and \# 451647/2015-7, and by FAPESP grant \# 2014/00304-2.

Received June 18, 2014; revised August 11, 2015. 
That is, there exists a continuous function $\varphi:(D \times U, D \times\{\mathbf{0}\}) \rightarrow\left(\mathbf{C}^{n}, \mathbf{0}\right)$ defined in a neighbourhood of the origin $(0, \mathbf{0}) \in \mathbf{C} \times \mathbf{C}^{n}$ such that, for all sufficiently small $t$, there is an open neighbourhood $U_{t} \subseteq U$ of $\mathbf{0} \in \mathbf{C}^{n}$ such that the map $\varphi_{t}:\left(U_{t}, \mathbf{0}\right) \rightarrow\left(\varphi\left(\{t\} \times U_{t}\right), \mathbf{0}\right)$ defined by $\varphi_{t}(\mathbf{z}):=\varphi(t, \mathbf{z})$ is a homeomorphism sending $V\left(f_{0}\right) \cap U_{t}$ onto $V\left(f_{t}\right) \cap \varphi_{t}\left(U_{t}\right)$. (We recall that a family of isolated singularities $\left\{f_{t}\right\}$ is said to be $\mu$-constant if, for all sufficiently small $t$, the Milnor number of $f_{t}$ at $\mathbf{0}$ is independent of $t$.) Under the same assumption, J. G. Timourian [18] showed that the family of functions $\left\{f_{t}\right\}$ itself is topologically trivial too-that is, $f_{0}=f_{t} \circ \varphi_{t}$ for all small $t$. In both cases, the restriction $n \neq 3$ comes from the use of the $h$-cobordism theorem. In [14], D. Massey asked whether the constancy of the Lê numbers $\lambda_{f_{t}, \mathbf{z}}^{i}(\mathbf{0})$ of $f_{t}$ at $\mathbf{0}$ with respect to the coordinates $\mathbf{z}$, as $t$ varies, is strong enough to imply that the local, ambient topological type of the hypersurfaces $V\left(f_{t}\right)$ remains constant in the case where $\left\{f_{t}\right\}$ is a family of non-isolated singularities. (We recall that the Lê numbers generalize to non-isolated singularities the data given by the Milnor number for an isolated singularity.) In [5], J. Fernández de Bobadilla answered positively this question when $n \geq 5$ and each $f_{t}$ has an 1-dimensional critical set-actually, he showed that the family of functions $\left\{f_{t}\right\}$ itself is topologically trivial. On the other hand, he showed in [4] that, in general, for higher-dimensional critical sets, the constancy of the Lê numbers $\lambda_{f_{t}, \mathbf{z}}^{i}(\mathbf{0})$ does not imply the constancy of the topological type, even for families which are linear in $t$ - that is, of the form $f_{t}(\mathbf{z})=f_{0}(\mathbf{z})+\operatorname{tg}(\mathbf{z})$, where $g:(U, \mathbf{0}) \rightarrow(\mathbf{C}, 0)$ is a holomorphic function. In [17], A. Parusiński proved that if $\left\{f_{t}\right\}$ is a $\mu$-constant linear deformation family of isolated singularities, then it is topologically trivial without any restriction on the integer $n$. Parusiński's approach does not use the $h$-cobordism theorem. His result is a consequence of Theorem 1.1 below_also due to Parusiński [17] —and a theorem of Lê Dũng Tráng and K. Saito [10] which says that, for a family of isolated singularities, the $t$-axis $D \times\{\mathbf{0}\}$ satisfies Thom's $a_{f}$ condition at the origin with respect to the ambient stratum if this family is $\mu$-constant.

THEOREM 1.1 (Parusiński). Suppose that $f$ is of the form $f(t, \mathbf{z})=f_{0}(\mathbf{z})+$ $\operatorname{tg}(\mathbf{z})$. If , in a neighbourhood of the origin,

$$
|g(\mathbf{z})| \ll\|\operatorname{grad} f(t, \mathbf{z})\|_{\infty} \quad \text { as }(t, \mathbf{z}) \rightarrow D \times\left(f_{0}^{-1}(0) \cap g^{-1}(0)\right),
$$

then the family $\left\{f_{t}\right\}$ is topologically trivial.

Note that, in this theorem, the singularities may be non-isolated.

As usual, grad $f(t, \mathbf{z})$ is the gradient vector of $f$ at $(t, \mathbf{z})$. If $h(t, \mathbf{z})$ denotes the real number $\|\operatorname{grad} f(t, \mathbf{z})\|_{\infty}$, then (1.1) means

$$
h^{-1}(0) \subseteq D \times g^{-1}(0),
$$

in a neighbourhood of $D \times\left(f_{0}^{-1}(0) \cap g^{-1}(0)\right)$, and

$$
\lim _{s \rightarrow 0} \frac{g \circ \gamma_{2}(s)}{h \circ \gamma(s)}=0
$$


for any holomorphic curve $\gamma: \mathbf{C} \rightarrow D \times U, s \mapsto \gamma(s):=\left(\gamma_{1}(s), \gamma_{2}(s)\right)$, not contained in the critical locus $\Sigma f$ of $f$ in a neighbourhood of $\gamma(0)$, with $\gamma(0) \in$ $D \times\left(f_{0}^{-1}(0) \cap g^{-1}(0)\right)$.

In the present paper, we show that any linear deformation $f$ of a (possibly non-isolated) hypersurface singularity $f_{0}$ is topologically trivial if the Lê numbers of $f$ are defined and constant along the strata of an analytic stratification of $D \times\left(f_{0}^{-1}(0) \cap g^{-1}(0)\right)$. Actually, it suffices the Lê numbers are constant along the strata that contain a point of $\Sigma f$. See Theorem 4.1 below. The proof is a combination of Parusiński's theorem (Theorem 1.1) and a generalization of the Lê-Saito theorem to non-isolated singularities due to D. Massey (cf. Theorem 3.2). But before to get to the heart of this paper, let us briefly remind the definition of the Lê numbers.

\section{Lê numbers}

The Lê numbers generalize to non-isolated hypersurface singularities the data given by the Milnor number for an isolated singularity. They were introduced about 25 years ago by $D$. Massey in $[12,13,14,15]$. They are intersection numbers of certain analytic cycles-so-called Lê cycles-with certain affine subspaces. As the definition of the Lê cycles involves the notion of gap sheaf, we shall start this section by explaining what 'analytic cycle' and 'gap sheaf' mean. We follow the presentation given in $[12,13,14,15]$.

2.1. Analytic cycles. Let $\left(X, \mathcal{O}_{X}\right)$ be a complex analytic space. An analytic cycle in $X$ is a finite formal sum $\sum m_{V}[V]$, where the $V$ 's are distinct irreducible analytic subsets of $X$ and the $m_{V}$ 's are non-zero integers. The analytic cycle associated to $X$ is the cycle $[X]:=\sum m_{V}[V]$ obtained when the $V$ 's run over all the irreducible components of $X$ and when the $m_{V}$ 's are defined as follows. Take a point $\mathbf{x} \in V$, and consider the germ $V_{\mathbf{x}}$ of $V$ at $\mathbf{x}$. Then, if $V_{\mathbf{x}}^{0}$ is any irreducible germ component of $V_{\mathbf{x}}$, the integer $m_{V}$ is defined to be the length of the local ring of $X$ at $\mathbf{x}$ localized at the prime ideal corresponding to $V_{\mathbf{x}}^{0}$. Of course, this definition is independent of the choices of the point $\mathbf{x}$ and the component $V_{\mathbf{x}}^{0}$. (Hereafter, we shall always use brackets [.] to denote analytic cycles.)

If $V$ and $W$ are irreducible analytic subsets of a connected complex analytic manifold $M$, then we say that $V$ and $W$ intersect properly in $M$ if, for each irreducible component $Z$ of $V \cap W$, we have:

$$
\operatorname{codim}_{M} Z=\operatorname{codim}_{M} V+\operatorname{codim}_{M} W .
$$

When this is the case, the intersection product of $[V]$ and $[W]$ in $M$ (denoted by $[V] \cdot[W])$ is characterized axiomatically by four properties: openness, transversality, projection and continuity (see [15, Appendix A] and [7, Example 11.4.4] for details). Now, if $\sum m_{i}\left[V_{i}\right]$ and $\sum n_{j}\left[W_{j}\right]$ are two cycles in $M$ intersecting 
properly (i.e., $V_{i}$ and $W_{j}$ intersect properly for all $i, j$ ), then we define their intersection product as

$$
\left(\sum m_{i}\left[V_{i}\right]\right) \cdot\left(\sum n_{j}\left[W_{j}\right]\right):=\sum m_{i} n_{j}\left(\left[V_{i}\right] \cdot\left[W_{j}\right]\right) .
$$

Finally, if $C_{1}$ and $C_{2}$ are two cycles intersecting properly and if $C_{1} \cdot C_{2}=$ $\sum p_{k}\left[Z_{k}\right]$, then the intersection number of $C_{1}$ and $C_{2}$ at $Z_{k}$ (denoted by $\left.\left(C_{1} \cdot C_{2}\right)_{Z_{k}}\right)$ is defined to be the integer $p_{k}$. In other words, $\left(C_{1} \cdot C_{2}\right)_{Z_{k}}$ represents the number of times $Z_{k}$ occurs in the intersection, counted with multiplicity.

2.2. Gap sheaves. Let again $\left(X, \mathcal{O}_{X}\right)$ be a complex analytic space, $W \subseteq X$ an analytic subset of $X$, and $\mathscr{I}$ a coherent sheaf of ideals in $\mathcal{O}_{X}$. As usual, we shall denote by $V(\mathscr{I})$ the analytic space defined by the vanishing of $\mathscr{I}$. At each point $\mathbf{x} \in V(\mathscr{I})$, we want to consider scheme-theoretically those components of $V(\mathscr{I})$ which are not contained in $W$. For this purpose, we look at a minimal primary decomposition of the stalk $\mathscr{I}_{\mathbf{x}}$ of $\mathscr{I}$ in the local ring $\mathcal{O}_{X, \mathbf{x}}$, and we consider the ideal $\mathscr{I}_{\mathbf{x}} \neg W$ in $\mathcal{O}_{X, \mathbf{x}}$ consisting of the intersection of those (possibly embedded) primary components $Q$ of $\mathscr{I}_{\mathbf{x}}$ such that $V(Q) \nsubseteq W$. If $S$ is the multiplicatively closed set

$$
\mathcal{O}_{X, \mathbf{x}} \backslash \bigcup P
$$

where the union is taken over all the associated prime ideals $P$ of $\mathcal{O}_{X, \mathbf{x}} / \mathscr{I}_{\mathbf{x}}$ such that $V(P) \nsubseteq W$, then

$$
\mathscr{I}_{\mathbf{x}} \neg W=S^{-1} \mathscr{I}_{\mathbf{x}} \cap \mathcal{O}_{X, \mathbf{x}},
$$

where $S^{-1} \mathscr{I}_{\mathbf{x}}$ is the ring of fractions of $\mathscr{I}_{\mathbf{x}}$ with denominators in $S$. In particular, the definition of $\mathscr{I}_{\mathbf{x}} \neg W$ does not depend on the choice of the minimal primary decomposition of $\mathscr{I}_{\mathbf{x}}$. Now, if we perform the operation described above at the point $\mathbf{x}$ simultaneously at all points of $V(\mathscr{I})$, then we obtain a coherent sheaf of ideals called a gap sheaf and denoted by $\mathscr{I} \neg W$. Hereafter, we shall denote by $V(\mathscr{I}) \neg W$ the scheme (i.e., the complex analytic space) $V(\mathscr{I} \neg W)$ defined by the vanishing of the gap sheaf $\mathscr{I} \neg W$.

2.3. Lê cycles and Lê numbers. Now, we are ready to define the Lê cycles and the Lê numbers. Consider a holomorphic function $h:(U, \mathbf{0}) \rightarrow(\mathbf{C}, 0)$, where $U$ is an open neighbourhood of $\mathbf{0}$ in $\mathbf{C}^{n}$, and fix a system of linear coordinates $\mathbf{z}=\left(z_{1}, \ldots, z_{n}\right)$ for $\mathbf{C}^{n}$. Let $\Sigma h$ be the critical locus of $h$. For $0 \leq i \leq n-1$, the $i$-th (relative) polar variety of $h$ with respect to $\mathbf{z}$ is the scheme

$$
\Gamma_{h, \mathbf{z}}^{i}:=V\left(\frac{\partial h}{\partial z_{i+1}}, \ldots, \frac{\partial h}{\partial z_{n}}\right) \neg \Sigma h .
$$

Clearly, for $i=0, \Gamma_{h, \mathbf{z}}^{0}=\emptyset$. Also, it can be shown easily that

$$
\left(\Gamma_{h, \mathbf{z}}^{i+1} \cap V\left(\frac{\partial h}{\partial z_{i+1}}\right)\right) \neg \Sigma h=\Gamma_{h, \mathbf{z}}^{i}
$$


as schemes, and therefore, all the components of the cycle

$$
\left[\Gamma_{h, \mathbf{z}}^{i+1} \cap V\left(\frac{\partial h}{\partial z_{i+1}}\right)\right]-\left[\Gamma_{h, \mathbf{z}}^{i}\right]
$$

are contained in $\Sigma h$. The cycle (2.1) is called the $i$-th Lê cycle of $h$ with respect to $\mathbf{z}$. It is denoted by $\left[\Lambda_{h, \mathbf{z}}^{i}\right]$.

DeFINITION 2.1. The $i$-th Lê number $\lambda_{h, \mathbf{z}}^{i}(\mathbf{p})$ of $h$ at $\mathbf{p}=\left(p_{1}, \ldots, p_{n}\right)$ with respect to the coordinates system $\mathbf{z}$ is defined to be the intersection number

$$
\lambda_{h, \mathbf{z}}^{i}(\mathbf{p}):=\left(\left[\Lambda_{h, \mathbf{z}}^{i}\right] \cdot\left[V\left(z_{1}-p_{1}, \ldots, z_{i}-p_{i}\right)\right]\right)_{\mathbf{p}},
$$

provided that this intersection is 0 -dimensional or empty at $\mathbf{p}$; otherwise, we say that $\lambda_{h, \mathbf{z}}^{i}(\mathbf{p})$ is undefined.

For $i=0$, the relation $(2.2)$ means

$$
\lambda_{h, \mathbf{z}}^{0}(\mathbf{p})=\left(\left[\Lambda_{h, \mathbf{z}}^{0}\right] \cdot U\right)_{\mathbf{p}}=\left[\Gamma_{h, \mathbf{z}}^{1} \cap V\left(\frac{\partial h}{\partial z_{1}}\right)\right]_{\mathbf{p}} .
$$

The last term is also equal to the intersection number

$$
\left(\left[\Gamma_{h, \mathbf{z}}^{1}\right] \cdot\left[V\left(\frac{\partial h}{\partial z_{1}}\right)\right]\right),
$$

whenever $\Gamma_{h, \mathbf{z}}^{1}$ is 1 -dimensional at $\mathbf{p}$.

For $\operatorname{dim}_{\mathbf{p}} \Sigma h<i \leq n-1, \lambda_{h, \mathbf{z}}^{i}(\mathbf{p})=0$. For this reason, we usually only consider the Lê numbers

$$
\lambda_{h, \mathbf{z}}^{0}(\mathbf{p}), \ldots, \lambda_{h, \mathbf{z}}^{\operatorname{dim}_{\mathbf{p}} \Sigma h}(\mathbf{p}) .
$$

If $\mathbf{p}$ is an isolated singularity of $h$, then $\lambda_{h, \mathbf{z}}^{0}(\mathbf{p})$ (which is the only possible non-zero Lê number) equals the Milnor number of $h$ at $\mathbf{p}$.

We conclude this section with the definition of the polar numbers.

Definition 2.2. The $i$-th polar number $\gamma_{h, \mathbf{z}}^{i}(\mathbf{p})$ of $h$ at $\mathbf{p}=\left(p_{1}, \ldots, p_{n}\right)$ with respect to the coordinates system $\mathbf{z}$ is defined to be the intersection number

$$
\gamma_{h, \mathbf{z}}^{i}(\mathbf{p}):=\left(\left[\Gamma_{h, \mathbf{z}}^{i}\right] \cdot\left[V\left(z_{1}-p_{1}, \ldots, z_{i}-p_{i}\right)\right]\right)_{\mathbf{p}},
$$

provided that this intersection is 0 -dimensional or empty at $\mathbf{p}$; otherwise, we say that $\gamma_{h, \mathbf{z}}^{i}(\mathbf{p})$ is undefined.

Note that $\gamma_{h, \mathbf{z}}^{0}(\mathbf{p})$ is always defined and equal to 0 .

Remark 2.3. For a generic choice of coordinates $\mathbf{z}$, for any point $\mathbf{p} \in V(h)$ near $\mathbf{0}$ and for any $0 \leq i \leq \operatorname{dim}_{\mathbf{p}} \Sigma h$, the Lê number $\lambda_{h, \mathbf{z}}^{i}(\mathbf{p})$ and the polar number $\gamma_{h, \mathbf{z}}^{i}(\mathbf{p})$ exist (cf. [14, Proposition 10.2 and Theorem 1.28]). 


\section{3. $\lambda_{(t, z)}$-constant linear deformations and Thom's $a_{f}$ condition}

Now, let us come back to the situation described in Section 1 , where $U$ is an open neighbourhood of $\mathbf{0}$ in $\mathbf{C}^{n}, D$ an open disc about 0 in $\mathbf{C},(t, \mathbf{z})$ linear coordinates for $\mathbf{C} \times \mathbf{C}^{n}$, and $f(t, \mathbf{z})$ a holomorphic function on $D \times U$ such that $f(t, \mathbf{0})=0$ and $\operatorname{dim}_{\mathbf{0}} \Sigma f_{t}$ is $\geq 0$ and constant as $t$ varies. Furthermore, in this section, we suppose that $f$ is linear in $t$, that is,

$$
f(t, \mathbf{z})=f_{0}(\mathbf{z})+\operatorname{tg}(\mathbf{z}) .
$$

By shrinking $D$ and $U$, we may assume $\Sigma f \subseteq f^{-1}(0)$. In particular, this implies $\Sigma f \subseteq D \times\left(f_{0}^{-1}(0) \cap g^{-1}(0)\right)$.

We fix an arbitrary analytic stratification $\mathscr{S}$ of $f_{0}^{-1}(0) \cap g^{-1}(0)$.

Definition 3.1. We say that the deformation $f$ is $\lambda_{(t, \mathbf{z})}$-constant with respect to the stratification $\mathscr{S}$ if, for any point $\mathbf{q}_{0}:=\left(a_{0}, \mathbf{p}_{0}\right)$ in the critical locus $\Sigma f$ of $f$ and for any integer $i, 0 \leq i \leq \operatorname{dim}_{\mathbf{q}_{0}} \Sigma f$, the $i$-th Lê number $\lambda_{f,(t, \mathbf{z})}^{i}(\mathbf{q})$ of $f$ at $\mathbf{q}$ with respect to the coordinates $(t, \mathbf{z})$ is defined and independent of $\mathbf{q}$ for all $\mathbf{q}:=(a, \mathbf{p}) \in D \times S\left(\mathbf{p}_{0}\right)$ near $\mathbf{q}_{0}$, where $S\left(\mathbf{p}_{0}\right)$ is the stratum of $\mathscr{S}$ containing $\mathbf{p}_{0}$.

In [14, Corollary 6.6], D. Massey proved the following theorem. (Actually, the statement given by Massey is much more general than the one given below, which is restricted to our situation.)

TheOREM 3.2 (Massey). If the deformation $f$ is $\lambda_{(t, \mathbf{z})}$-constant with respect to the stratification $\mathscr{S}$, then, for any $\mathbf{q}_{0}:=\left(a_{0}, \mathbf{p}_{0}\right) \in \Sigma f$, the submanifold $D \times S\left(\mathbf{p}_{0}\right)$ of $D \times\left(f_{0}^{-1}(0) \cap g^{-1}(0)\right)$ containing $\mathbf{q}_{0}$ satisfies Thom's $a_{f}$ condition at $\mathbf{q}_{0}$ with respect to the ambient stratum. That is, if $\left\{\mathbf{q}_{k}\right\}$ is a sequence of points in $(D \times U) \backslash \Sigma f$ such that

$$
\mathbf{q}_{k} \rightarrow \mathbf{q}_{0} \quad \text { and } \quad T_{\mathbf{q}_{k}} V\left(f-f\left(\mathbf{q}_{k}\right)\right) \rightarrow T,
$$

then $T_{\mathbf{q}_{0}}\left(D \times S\left(\mathbf{p}_{0}\right)\right) \subseteq T$.

As usual, $T_{\mathbf{q}_{k}} V\left(f-f\left(\mathbf{q}_{k}\right)\right)$ denotes the tangent space at $\mathbf{q}_{k}$ to the level hypersurface in $\mathbf{C} \times \mathbf{C}^{n}$ defined by $f(t, \mathbf{z})=f\left(\mathbf{q}_{k}\right)$, and $T_{\mathbf{q}_{0}}\left(D \times S\left(\mathbf{p}_{0}\right)\right)$ the tangent space at $\mathbf{q}_{0}=\left(a_{0}, \mathbf{p}_{0}\right)$ to $D \times S\left(\mathbf{p}_{0}\right)$.

LEMMA 3.3. Let $\mathbf{q}_{0}:=\left(a_{0}, \mathbf{p}_{0}\right)$ be any point in $\Sigma f$, and let $S\left(\mathbf{p}_{0}\right)$ be the stratum of $\mathscr{S}$ containing $\mathbf{p}_{0}$. If $D \times S\left(\mathbf{p}_{0}\right)$ satisfies Thom's $a_{f}$ condition at $\mathbf{q}_{0}$ with respect to the ambient stratum, then

$$
|g(\mathbf{z})| \ll\|\operatorname{grad} f(t, \mathbf{z})\|_{\infty} \quad \text { as }(t, \mathbf{z}) \rightarrow\left(a_{0}, \mathbf{p}_{0}\right) .
$$

Proof. First, we observe that if $D \times S\left(\mathbf{p}_{0}\right)$ satisfies Thom's $a_{f}$ condition at $\mathbf{q}_{0}$ with respect to the ambient stratum, then, for any holomorphic curve

$$
\gamma:(\mathbf{C}, 0) \rightarrow\left(D \times U, \mathbf{q}_{0}\right), \quad s \mapsto \gamma(s):=\left(\gamma_{1}(s), \gamma_{2}(s)\right)
$$


not contained in $\Sigma f$ in an arbitrarily small neighbourhood of $\mathbf{q}_{0}$, we have

$$
\operatorname{ord}\left(\frac{\partial f}{\partial t} \circ \gamma\right)>\inf _{1 \leq i \leq n} \operatorname{ord}\left(\frac{\partial f}{\partial z_{i}} \circ \gamma\right),
$$

where ord $(\cdot)$ is the order in $s$ at 0 . To show this, we proceed as in [3, Lemma 2.2]. Suppose there is a holomorphic curve $\gamma$ for which the condition (3.1) does not hold. Then, the limit, as $s \rightarrow 0$, of the projective class of

$$
\operatorname{grad} f(\gamma(s))=\left(\frac{\partial f}{\partial t}(\gamma(s)), \frac{\partial f}{\partial z_{1}}(\gamma(s)), \ldots, \frac{\partial f}{\partial z_{n}}(\gamma(s))\right)
$$

has the form $\left[w: v_{1}: \cdots: v_{n}\right]$ with $w \neq 0$, and therefore $D \times S\left(\mathbf{p}_{0}\right)$ does not satisfy Thom's $a_{f}$ condition along the curve $\gamma-$ a contradiction. Now, as $f$ is linear in $t$,

$$
\frac{\partial f}{\partial t} \circ \gamma(s)=g \circ \gamma_{2}(s)
$$

and hence, by (3.1),

$$
\left|g \circ \gamma_{2}(s)\right| \ll\|\operatorname{grad} f(\gamma(s))\|_{\infty} \quad \text { as } s \rightarrow 0 .
$$

This completes the proof of Lemma 3.3.

\section{Topological triviality of $\lambda_{(t, z)}$-constant linear deformations}

We still assume that $f$ is of the form $f(t, \mathbf{z})=f_{0}(\mathbf{z})+\operatorname{tg}(\mathbf{z})$, and we still denote by $\mathscr{S}$ any given analytic stratification of $f_{0}^{-1}(0) \cap g^{-1}(0)$.

THEOREM 4.1. Under the above assumptions, if $f$ is $\lambda_{(t, \mathbf{z})}$-constant with respect to the stratification $\mathscr{S}$, then the family $\left\{f_{t}\right\}$ is topologically trivial.

Proof. By Theorem 1.1, it suffices to show that, for any $\mathbf{q} \in D \times\left(f_{0}^{-1}(0) \cap\right.$ $\left.g^{-1}(0)\right)$,

$$
|g(\mathbf{z})| \ll\|\operatorname{grad} f(t, \mathbf{z})\|_{\infty} \quad \text { as }(t, \mathbf{z}) \rightarrow \mathbf{q} .
$$

By Theorem 3.2 and Lemma 3.3, this is true for any point $\mathbf{q}$ in $\Sigma f$. Actually, this is also true for any point $\mathbf{q} \in\left(D \times\left(f_{0}^{-1}(0) \cap g^{-1}(0)\right)\right) \backslash \Sigma f$. Indeed, for such a point $\mathbf{q}=(a, \mathbf{p})$, there exist an index $i_{0}, 1 \leq i_{0} \leq n$, such that $\left(\partial f / \partial z_{i_{0}}\right)(\mathbf{q}) \neq 0$, while $(\partial f / \partial t)(\mathbf{q})=g(\mathbf{p})=0$.

To show that Theorem 4.1 is not vacuously true, let us give an example where its assumptions are satisfied. Let $U_{1}$ be an open neighbourhood of $\mathbf{0} \in \mathbf{C}^{3}$, $D$ an open disc about $0 \in \mathbf{C}$, and let $F: D \times U_{1} \rightarrow \mathbf{C}$ be the Briançon-Speder example [1] - that is, the family of isolated singularities defined by

$$
(t, \mathbf{z}):=\left(t, z_{1}, z_{2}, z_{3}\right) \mapsto F(t, \mathbf{z}):=z_{3}^{5}+z_{2}^{7} z_{1}+z_{1}^{15}+t z_{2}^{6} z_{3} .
$$


Now, pick an open neighbourhood $U_{2}$ of $0 \in \mathbf{C}$ and consider the function $f: D \times U_{2} \times U_{1} \rightarrow \mathbf{C}$ defined by

$$
(t, w, \mathbf{z}) \mapsto f(t, w, \mathbf{z}):=F(t, \mathbf{z}) .
$$

This example is already used in $[11, \S 5]$. Clearly, the family $\left\{f_{t}\right\}$ defined by $f$ (i.e., the family of functions given by $\left.f_{t}(w, \mathbf{z}):=f(t, w, \mathbf{z})\right)$ is a family of line singularities, and we have $\Sigma f=D \times U_{2} \times\{\boldsymbol{0}\}$ and $\Sigma f_{t}=U_{2} \times\{\boldsymbol{0}\}$.

By [14, Proposition 10.2 and Theorem 1.28], for a generic choice of linear coordinates $(t, w, \mathbf{z})$, for any point $(a, b, \mathbf{p}) \in V(f)$ in a neighbourhood of the origin and for any $0 \leq i \leq \operatorname{dim}_{(a, b, \mathbf{p})} \Sigma f=2$, the Lê number $\lambda_{f,(t, w, \mathbf{z})}^{i}(a, b, \mathbf{p})$ and the polar number $\gamma_{f,(t, w, \mathbf{z})}^{i}(a, b, \mathbf{p})$ exist. Hereafter, we fix such a generic coordinates system. Then, as $\Sigma f \cap\left(\{a\} \times U_{2} \times U_{1}\right)=\{a\} \times \Sigma f_{a}$, Proposition 1.21 of [14] applies and shows that

$$
\left\{\begin{array}{l}
\lambda_{f_{a},(w, \mathbf{z})}^{1}(b, \mathbf{p})=\lambda_{f,(t, w, \mathbf{z})}^{2}(a, b, \mathbf{p}), \\
\gamma_{f_{a},(w, \mathbf{z})}^{1}(b, \mathbf{p})=\gamma_{f,(t, w, \mathbf{z})}^{2}(a, b, \mathbf{p}), \\
\lambda_{f_{a},(w, \mathbf{z})}^{0}(b, \mathbf{p})=\gamma_{f,(t, w, \mathbf{z})}^{1}(a, b, \mathbf{p})+\lambda_{f,(t, w, \mathbf{z})}^{1}(a, b, \mathbf{p}) .
\end{array}\right.
$$

Furthermore, as $\Sigma f_{a} \cap\left(\{b\} \times U_{1}\right)=\{b\} \times \Sigma\left(f_{a}\right)_{b} \quad$ and $\quad$ as $\quad \lambda_{f_{a},(w, \mathbf{z})}^{i}(b, \mathbf{p})$ and $\gamma_{f_{a},(w, \mathbf{z})}^{i}(b, \mathbf{p})$ exist for any $(b, \mathbf{p}) \in V\left(f_{a}\right)$ in a neighbourhood of the origin and for any $0 \leq i \leq 1$, Proposition 1.21 of [14] also shows that

$$
\lambda_{\left(f_{a}\right)_{b}, \mathbf{z}}^{0}(\mathbf{p})=\gamma_{f_{a},(w, \mathbf{z})}^{1}(b, \mathbf{p})+\lambda_{f_{a},(w, \mathbf{z})}^{1}(b, \mathbf{p}),
$$

where $\left(f_{a}\right)_{b}(\mathbf{z}):=f_{a}(b, \mathbf{z})=F_{a}(\mathbf{z})$. Since $F_{a}$ has an isolated singularity at the origin, the Lê number $\lambda_{\left(f_{a}\right)_{b}, \mathbf{z}}^{0}(\mathbf{0})$ is nothing but the Milnor number of $F_{a}$ at $\mathbf{0}$, which is constant as $a$ varies. (Note that $\lambda_{\left(f_{a}\right)_{b}, \mathbf{z}}^{0}(\mathbf{p})=0$ for $\mathbf{p} \neq \mathbf{0}$.)

Now, as the Briançon-Speder family $\left\{F_{t}\right\}$ is $\mu$-constant, the $t$-axis $D \times\{\mathbf{0}\}$ satisfies Thom's $a_{F}$ condition at $(0, \mathbf{0})$ with respect to the ambient stratum $\left(D \times U_{1}\right) \backslash \Sigma F$ (cf. [10]). Then, the proof of Lemma 44 of [5] shows that, in a neighbourhood of the origin in $D \times U_{1}$,

$$
\Sigma F:=V\left(\frac{\partial F}{\partial t}, \frac{\partial F}{\partial z_{1}}, \ldots, \frac{\partial F}{\partial z_{n}}\right)=V\left(\frac{\partial F}{\partial z_{1}}, \ldots, \frac{\partial F}{\partial z_{n}}\right) .
$$

This, in turn, implies that, in a neighbourhood of the origin in $D \times U_{2} \times U_{1}$,

$$
\Sigma f=V\left(\frac{\partial f}{\partial w}, \frac{\partial f}{\partial z_{1}}, \ldots, \frac{\partial f}{\partial z_{n}}\right) .
$$

It follows that the 1-st polar variety $\Gamma_{f,(t, w, \mathbf{z})}^{1}$ is empty, and hence,

$$
\gamma_{f,(t, w, \mathbf{z})}^{1}(a, b, \mathbf{p}):=\left(\left[\Gamma_{f,(t, w, \mathbf{z})}^{1}\right] \cdot[V(t-a)]\right)_{(a, b, \mathbf{p})}=0
$$


and

$$
\lambda_{f,(t, w, \mathbf{z})}^{0}(a, b, \mathbf{p}):=\left[\Gamma_{f,(t, w, \mathbf{z})}^{1} \cap V\left(\frac{\partial f}{\partial t}\right)\right]_{(a, b, \mathbf{p})}=0,
$$

for any $(a, b, \mathbf{p}) \in V(f)$. Moreover, as

$$
\Sigma f_{a}=V\left(\frac{\partial f_{a}}{\partial z_{1}}, \ldots, \frac{\partial f_{a}}{\partial z_{n}}\right) \subseteq U_{2} \times U_{1}
$$

$\Gamma_{f_{a},(w, \mathbf{z})}^{1}$ is empty, and hence,

$$
\gamma_{f_{a},(w, \mathbf{z})}^{1}(b, \mathbf{p})=\lambda_{f_{a},(w, \mathbf{z})}^{0}(b, \mathbf{p})=0,
$$

for any $(b, \mathbf{p}) \in V\left(f_{a}\right)$.

Combining $(4.1)-(4.5)$ gives $\lambda_{f,(t, w, \mathbf{z})}^{0}(a, b, \mathbf{p})=\lambda_{f,(t, w, \mathbf{z})}^{1}(a, b, \mathbf{p})=0$ and

$$
\lambda_{f,(t, w, \mathbf{z})}^{2}(a, b, \mathbf{p})=\left\{\begin{array}{l}
\mu_{F_{a}}(\mathbf{0}) \quad \text { if }(a, b, \mathbf{p}) \in \Sigma f, \\
0 \quad \text { if }(a, b, \mathbf{p}) \in V(f) \backslash \Sigma f,
\end{array}\right.
$$

where $\mu_{F_{a}}(\mathbf{0})$ is the Milnor number of $F_{a}$ at $\mathbf{0}$. It follows that the deformation $f$ is $\lambda_{(t, w, \mathbf{z})}$-constant with respect to the analytic stratification of $f_{0}^{-1}(0) \cap g^{-1}(0)$ consisting in the following two strata: $U_{2} \times\{\boldsymbol{0}\}$ and $\left(f_{0}^{-1}(0) \cap g^{-1}(0)\right) \backslash\left(U_{2} \times\{\boldsymbol{0}\}\right)$. (Here, $f_{0}(w, \mathbf{z})=z_{3}^{5}+z_{2}^{7} z_{1}+z_{1}^{15}$ and $g(w, \mathbf{z})=z_{2}^{6} z_{3}$.) Therefore, Theorem 4.1 applies. (Of course, in this special case, since the family $\left\{F_{t}\right\}$ is topologically trivial, we knew from the beginning that the family $\left\{f_{t}\right\}$ must be topologically trivial too.)

By the Thom-Mather first isotopy theorem $[16,19]$, we know that if $h(t, \mathbf{z})$ is a holomorphic function in a neighbourhood of the origin in $\mathbf{C} \times \mathbf{C}^{n}$ and if there exists a Whitney stratification of the hypersurface $V(h)$ such that the $t$-axis can be chosen as a stratum, then the family of hypersurfaces $\left\{V\left(h_{t}\right)\right\}$ is topologically trivial. The Briançon-Speder family $\left\{F_{t}\right\}$ is a topologically trivial family such that the $t$-axis (in $D \times U_{1}$ ) cannot be chosen as a Whitney stratum of $V(F)$ (cf. [1]). As it is explained by D. Massey in [11, §5], the $t$-axis (in $D \times U_{2} \times U_{1}$ ) cannot be chosen as a Whitney stratum of $V(f)$ either, where $f$ is as in the above example. Thus, this example shows that the assumptions in Theorem 4.1 are not so strong in the sense that they do not imply the Whitney conditions along the $t$-axis.

\section{5. $\lambda_{(t, \mathrm{z})}$-constancy along the $t$-axis}

Now, let us come back to the general setting described in Section 3 and at the beginning of Section 4 . If $f$ is $\lambda_{(t, \mathbf{z})}$-constant with respect to $\mathscr{S}$, then, clearly, it is also $\lambda_{(t, \mathbf{z})}$-constant along the $t$-axis in a neighbourhood of the origin-that is, for all $0 \leq i \leq \operatorname{dim}_{(0,0)} \Sigma f$, the $i$-th Lê number $\lambda_{f,(t, \mathbf{z})}^{i}(a, \mathbf{0})$ of $f$ at $(a, \mathbf{0})$ with respect to the coordinates $(t, \mathbf{z})$ is defined and independent of $a$ for all $a$ near 0 . Obviously, the converse is not true. Then, we may ask what is the relationship 
between the $\lambda_{(t, \mathbf{z})}$-constancy along the $t$-axis in a neighbourhood of the origin and the constancy of the Lê numbers $\lambda_{f, \mathbf{z}}^{i}(\mathbf{0})$ of $f_{t}$ at $\mathbf{0}$, with respect to the coordinates $\mathbf{z}$, as $t$ varies. It turns out that they are equivalent. Essentially, this is proved in [14, Proposition 1.21], [5, Lemma 44 and the relations (60) and (61)] and [2, Theorem 4.1 and Corollary 4.2].

THEOREM 5.1. Suppose that, for all a near 0 and all $0 \leq i \leq \operatorname{dim}_{(a, \mathbf{0})} \Sigma f$, the Lê number $\lambda_{f,(t, \mathbf{z})}^{i}(a, \mathbf{0})$ and the polar number $\gamma_{f,(t, \mathbf{z})}^{i}(a, \mathbf{0})$ exist. Then, the following two assertions are equivalent.

(1) The deformation $f$ is $\lambda_{(t, \mathbf{z})}$-constant along the t-axis in a neighbourhood of the origin.

(2) The family $\left\{f_{t}\right\}$ is $\lambda_{\mathbf{z}}$-constant.

Here, we say that $\left\{f_{t}\right\}$ is $\lambda_{\mathbf{z}}$-constant if for all $0 \leq i \leq \operatorname{dim}_{\mathbf{0}} \Sigma f_{0}=\operatorname{dim}_{\mathbf{0}} \Sigma f_{t}$, the $i$-th Lê number $\lambda_{f_{t}, \mathbf{z}}^{i}(\mathbf{0})$ is defined and independent of $t$, for all $t$ near 0 .

In this theorem, we do not assume that $f$ is linear in $t$.

Note that, by [14, Proposition 10.2 and Theorem 1.28], for a generic choice of coordinates $(t, \mathbf{z})$, for any $a$ near 0 and for any $0 \leq i \leq \operatorname{dim}_{(a, 0)} \Sigma f$, the Lê number $\lambda_{f,(t, \mathbf{z})}^{i}(a, \mathbf{0})$ and the polar number $\gamma_{f,(t, \mathbf{z})}^{i}(a, \mathbf{0})$ exist.

Theorem 5.1 is not essential for our purpose. However, it may help to understand why the $\lambda_{\mathrm{z}}$-constancy is not enough to get the topological triviality. To obtain the topological triviality, we need to control the Lê numbers not only on the $t$-axis but also all around $\Sigma f$ within $D \times\left(f_{0}^{-1}(0) \cap g^{-1}(0)\right)$.

Proof of Theorem 5.1. If $f$ is $\lambda_{(t, \mathbf{z})}$-constant along the $t$-axis in a neighbourhood of the origin, then, by [14, Corollary 6.6], the $t$-axis $D \times\{\mathbf{0}\}$ satisfies Thom's $a_{f}$ condition at the origin with respect to the ambient stratum. Hence, the proof of Lemma 44 of [5] shows that, in a neighbourhood of the origin,

$$
\Sigma f:=V\left(\frac{\partial f}{\partial t}, \frac{\partial f}{\partial z_{1}}, \ldots, \frac{\partial f}{\partial z_{n}}\right)=V\left(\frac{\partial f}{\partial z_{1}}, \ldots, \frac{\partial f}{\partial z_{n}}\right) .
$$

In particular, for all $a$ small enough, $\Sigma f \cap\left(\{a\} \times \mathbf{C}^{n}\right)=\{a\} \times \Sigma f_{a}$, and hence, by [14, Propositions 1.16 and 1.21],

$$
\left\{\begin{array}{l}
\lambda_{f_{a}, \mathbf{z}}^{i}(\mathbf{0})=\lambda_{f,(t, \mathbf{z})}^{i+1}(a, \mathbf{0}) \quad \text { for } 1 \leq i \leq \operatorname{dim}_{(a, \mathbf{0})} \Sigma f-1=\operatorname{dim}_{\mathbf{0}} \Sigma f_{a}, \\
\lambda_{f_{a}, \mathbf{z}}^{0}(\mathbf{0})=\gamma_{f,(t, \mathbf{z})}^{1}(a, \mathbf{0})+\lambda_{f,(t, \mathbf{z})}^{1}(a, \mathbf{0}) .
\end{array}\right.
$$

Furthermore, (5.1) also shows that the 1-st polar variety $\Gamma_{f,(t, \mathbf{z})}^{1}$ is empty. Therefore,

$$
\gamma_{f,(t, \mathbf{z})}^{1}(a, \mathbf{0}):=\left(\left[\Gamma_{f,(t, \mathbf{z})}^{1}\right] \cdot[V(t-a)]\right)_{(a, \mathbf{0})}=0 .
$$

It follows immediately that the family $\left\{f_{t}\right\}$ is $\lambda_{\mathbf{z}}$-constant.

Conversely, if the family $\left\{f_{t}\right\}$ is $\lambda_{\mathbf{z}}$-constant, then, by [14, Theorem 6.5], the $t$-axis satisfies Thom's $a_{f}$ condition at the origin with respect to the ambient 
stratum. Hence, as above, (5.1) holds and $\Gamma_{f,(t, \mathbf{z})}^{1}=\emptyset$. Therefore, for all $a$ sufficiently small,

$$
\gamma_{f,(t, \mathbf{z})}^{1}(a, \mathbf{0})=0
$$

and

$$
\lambda_{f,(t, \mathbf{z})}^{0}(a, \mathbf{0}):=\left[\Gamma_{f,(t, \mathbf{z})}^{1} \cap V\left(\frac{\partial f}{\partial t}\right)\right]_{(a, \mathbf{0})}=0 .
$$

Furthermore, Propositions 1.16 and 1.21 of [14] apply and show that (5.2) holds. It follows that $f$ is $\lambda_{(t, \mathbf{z})}$-constant along the $t$-axis in a neighbourhood of the origin.

As mentioned in the introduction, J. Fernández de Bobadilla gave in [4] an example of a $\lambda_{\mathbf{z}}$-constant family which is not topologically trivial. This family is given by

$$
f\left(t, z_{1}, \ldots, z_{5}\right):=z_{3} z_{4}^{2}-z_{3} z_{5}^{2}+2 z_{2} z_{4} z_{5}+t z_{1} z_{5}^{2} .
$$

Let us show that this family does not satisfy the hypotheses of Theorem 4.1. More precisely, if $\mathbf{p}_{0}:=\left(p_{1}, 0,0,0,0\right)$ with $p_{1} \neq 0$, then we claim that for any analytic stratification $\mathscr{S}$ of $f_{0}^{-1}(0) \cap g^{-1}(0)$,

$$
\lambda_{f,(t, \mathbf{z})}^{3}\left(0, \mathbf{p}_{0}\right) \neq \lambda_{f,(t, \mathbf{z})}^{3}\left(a, \mathbf{p}_{0}\right)
$$

for any $a \neq 0$, where $f_{0}\left(z_{1}, \ldots, z_{5}\right)=z_{3} z_{4}^{2}-z_{3} z_{5}^{2}+2 z_{2} z_{4} z_{5}$ and $g\left(z_{1}, \ldots, z_{5}\right)=$ $z_{1} z_{5}^{2}$. In particular, $f$ is not $\lambda_{(t, \mathbf{z})}$-constant with respect to $\mathscr{S}$. To compute the Lê numbers (5.3), we first observe that the 4-th and 3-rd polar varieties of $f$ with respect to the coordinates $(t, \mathbf{z})$ are given by

$$
\begin{aligned}
& \Gamma_{f,(t, \mathbf{z})}^{4}=V\left(z_{3} z_{4}+z_{2} z_{5}, z_{2} z_{4}+z_{5}\left(t z_{1}-z_{3}\right), z_{3}\left(t z_{1}-z_{3}\right)-z_{2}^{2}\right), \\
& \Gamma_{f,(t, \mathbf{z})}^{3}=V\left(2 z_{2}+t z_{1}, z_{3}+z_{2}, z_{4}-z_{5}\right) \cup V\left(2 z_{2}-t z_{1}, z_{3}-z_{2}, z_{4}+z_{5}\right) .
\end{aligned}
$$

Thus the 3-rd Lê cycle of $f$ with respect to $(t, \mathbf{z})$ is given by

$$
\left[\Lambda_{f,(t, \mathbf{z})}^{3}\right]=2\left[V\left(z_{4}, z_{5}, z_{3}\left(t z_{1}-z_{3}\right)-z_{2}^{2}\right)\right] .
$$

It follows that $\lambda_{f,(t, \mathbf{z})}^{3}\left(0, \mathbf{p}_{0}\right)=4$ while $\lambda_{f,(t, \mathbf{z})}^{3}\left(a, \mathbf{p}_{0}\right)=2$ for any $a \neq 0$.

To conclude, let us mention some interesting features of $\lambda_{\mathbf{z}}$-constant families. Certainly, the most important one, due to D. Massey [13, 14, 15], says that, for a generic choice of coordinates $\mathbf{z}$, if the family $\left\{f_{t}\right\}$ is $\lambda_{\mathbf{z}}$-constant and if $\operatorname{dim}_{0} \Sigma f_{t} \leq n-4$, then the diffeomorphism type of the Milnor fibration of $f_{t}$ at the origin is independent of $t$, for all $t$ small. (When $\operatorname{dim}_{0} \Sigma f_{t}=0$-in which case the $\lambda_{\mathbf{z}}$-constancy is equivalent to the $\mu$-constancy-the result was proved by Lê Dũng Tráng and C. P. Ramanujam [9].)

Finally, related to the Zariski multiplicity conjecture [21] for non-isolated singularities, the authors showed in [3] that if $f$ is linear in $t$ and if the family 
$\left\{f_{t}\right\}$ is $\lambda_{\mathbf{z}}$-constant, then the order of $f_{t}$ at $\mathbf{0}$ is independent of $t$, for all small $t$. (When $\operatorname{dim}_{0} \Sigma f_{t}=0$, the result was first proved by G.-M. Greuel [8] and D. Trotman [20].)

Acknowledgments. The authors thank the referee for valuable comments and suggestions that led to improvements in this paper.

\section{REFERENCES}

[1] J. BRiançon AND J.-P. SPEDER, La trivialité topologique n'implique pas les conditions de Whitney, C. R. Acad. Sci. Paris, Sér. A-B. 280 (1975), 365-367 (in French).

[2] R. Callejas-Bedregal, V. H. Jorge Pérez, M. J. Saia and J. N. Tomazella, Lê constant families of singular hypersurfaces, Proyecciones 31 (2012), 333-343.

[3] C. Eyral and M. Ruas, Deformations with constant Lê numbers and multiplicities of non-isolated hypersurface singularities, Nagoya Math. J. 218 (2015), 29-50.

[4] J. Fernández de Bobadilla, Answers to some equisingularity questions, Invent. Math. 161 (2005), 657-675.

[5] J. Fernández de Bobadilla, Topological equisingularity of hypersurfaces with 1-dimensional critical set, Adv. Math. 248 (2013), 1199-1253.

[6] J. Fernández de Bobadilla and T. Gaffney, The Lê numbers of the square of a function and their applications, J. Lond. Math. Soc. (2) 77 (2008), 545-557.

[ 7 ] W. Fulton, Intersection theory, Ergebnisse der Mathematik und ihrer Grenzgebiete (3) 2, Springer-Verlag, Berlin, 1984.

[ 8 ] G.-M. GreuEL, Constant Milnor number implies constant multiplicity for quasihomogeneous singularities, Manuscripta Math. 56 (1986), 159-166.

[9] Lê Dũng Tráng and C. P. Ramanujam, The invariance of Milnor number implies the invariance of the topological type, Amer. J. Math. 98 (1976), 67-78.

[10] Lê Dũng Tráng and K. SaIto, La constance du nombre de Milnor donne des bonnes stratifications, C. R. Acad. Sci. Paris, Sér. A-B. 277 (1973), 793-795 (in French).

[11] D. MASSEY, The Lê-Ramanujam problem for hypersurfaces with one-dimensional singular sets, Math. Ann. 282 (1988), 33-49.

[12] D. Massey, The Lê varieties, I, Invent. Math. 99 (1990), 357-376.

[13] D. Massey, The Lê varieties, II, Invent. Math. 104 (1991), 113-148.

[14] D. MAssey, Lê cycles and hypersurface singularities, Lecture notes in math. 1615, SpringerVerlag, Berlin, 1995.

[15] D. Massey, Numerical control over complex analytic singularities, Mem. Amer. Math. Soc. 163 (2003), no. 778.

[16] J. Mather, Notes on topological stability, Bull. Amer. Math. Soc. (N. S.) 49 (2012), 475-506.

[17] A. PARUsińsKi, Topological triviality of $\mu$-constant deformations of type $f(x)+\operatorname{tg}(x)$, Bull. London Math. Soc. 31 (1999), 686-692.

[18] J. G. Timourian, The invariance of Milnor's number implies topological triviality, Amer. J. Math. 99 (1977), 437-446.

[19] R. Tном, Ensembles et morphismes stratifiés, Bull. Amer. Math. Soc. 75 (1969), 240-284 (in French).

[20] D. Trotman, Partial results on the topological invariance of the multiplicity of a complex hypersurface, the A'Campo-MacPherson Seminar, Univ. Paris VII, 1977, available in: D. Trotman, Équisingularité et conditions de Whitney, Thèse d'état, Univ. Paris XI, 1980. 
TOPOLOGICAL TRIVIALITY OF LINEAR DEFORMATIONS WITH CONSTANT LÊ NUMBERS 201

[21] O. Zariski, Some open questions in the theory of singularities, Bull. Amer. Math. Soc. 77 (1971), 481-491.

Christophe Eyral

INSTYTUT MATEMATYCZNY

Polskiej Akademit Nauk

UL. ŚNIADECKich 8, 00-656 Warszawa

PolAND

E-mail: eyralchr@yahoo.com

Maria Aparecida Soares Ruas

Instituto de Ciências Matemáticas e de Computação

Universidade de São Paulo

Avenida Trabalhador São-Carlense

400-Centro, Caixa Postal 668

13566-590 SÃo CARLOS-SP

BRAZIL

E-mail: maasruas@icmc.usp.br 